\title{
Rate Based Congestion Control for Wireless Links in Information Centric Network
}

\author{
Mahesh R. Patil, L. Agilandeeswari
}

\begin{abstract}
Information-centric networking (ICN) is the prominent network architecture with the features of name based forwarding and in-network caching. These features enables ICN to provide solutions to all demands of the emerging networks. Among the proposed hop by hop congestion control schemes in ICN, almost all schemes assume the available link capacity as known and fixed which is not true for wireless links. Here we propose dynamic link capacity estimation for wireless links using kalman filter and each node maintains the data rate value estimated. Consumer forwards interest with initial data rate, each hop updates estimated data rate in outgoing data packets proactively, then consumer adjusts the data rate according to new value received.
\end{abstract}

\section{Keywords: Congestion Control, ICN, NDN, ndnSIM}

\section{INTRODUCTION}

Information centric network (ICN) is a kind of network structure which is data centric rather than host centric, i.e. in this type of network more importance is given to the content being carried on network rather than from where the content is extracted. In legacy networks content is delivered from origin servers, but here in ICN it's not necessary that content to be delivered from origin servers, it can be delivered from anywhere in the network because this content is location independent. Introduction of in-network caching feature in ICN enables the routers to store content in their content store, when a request arrives for a particular content, router checks whether the content is available in its store, if it is available then router responds to that request by sending content back to the requester. Research efforts in ICN are wide spread among the areas of naming, routing, congestion control, security and privacy etc. Article [1] gives a brief introduction about ICN, features of ICN and it summarizes the post architectural work done in the areas of naming, routing, congestion control and security, among these areas there has been less research done in the area of congestion control. There are various proposals in congestion control like hop by hop congestion control, end to end congestion control, and multi-path and multisource congestion control. Some of them are rate based congestion control and some are window based congestion control. Most of these proposals consider path links with static bandwidth which is true for wired

Revised Manuscript Received on December 02, 2019

* Correspondence Author

Mahesh R. Patil*, School of Information Technology and Engineering, Vellore Institute of Technology, Vellore, India.

Email: maheshpatil67@gmail.com

L. Agilandeeswari, School of Information Technology and Engineering, Vellore Institute of Technology, Vellore, India. Email: agila.1@ vit.ac.in networks but not for wireless networks. Link bandwidth for wireless links tend to change periodically and the existing proposals are optimized only for wired links or the links whose bandwidth does not change periodically. In [2] the authors proposed a rate based congestion control protocol for wireless links, in which a simple rate control protocol is combined with wireless link bandwidth estimation to know the true available bandwidth in the network path and intimate the consumer about the same. But this approach is reactive to congestion where consumer or in-network nodes react to congestion triggered NACKs, and decrease the interest sending rate. Although there is need of more sophisticated rate based congestion control to notify the consumer or any in-network node proactively. One of such proactive multipath aware rate based congestion control (MIRCC) is proposed by Milad. M [3], here when producer receives interest from consumer it responds back with data packets, it adds data sending rate $R(t)$ in data packet header which is calculated by MIRCC logic. In-network nodes maintain the database of adjacent link bandwidth. When data packet arrives at in-network node then this node reads $R(t)$ embedded in the data packet header and if this $R(t)$ value is greater than the value which it holds, then it updates $R(t)$ with smaller value, later this packet is delivered to consumer. Consumer adjusts the interest sending rate according to new $R(t)$ value. But even this proposal considers all path links with fixed bandwidth. So there is need of proactive rate notification of actual available bandwidth and also must be tuned for wireless networks.

This article is about proactive rate notification to consumer through MIRCC logic as well as it applies kalman filter to get the available wireless link bandwidth continuously so that the true value of $R(t)$ is delivered to consumer/in-network router. The remaining paper is organized in following manner; in section 2 there is summarization of related work, in section 3 single path MIRCC is introduced, in section 4 our proposed architecture Modified MIRCC is described, in section 5 information about the simulation setup is given, section 6 describes the simulation results and at the end section 7 concludes this paper.

\section{RELATED WORK}

In article [4] authors have proposed estimating the end to end available bandwidth of a network path in real time. Here a small amount of probe packet pairs are induced into the network at random rates, to measure the available end to end bandwidth. This end to end bandwidth is measured by the effect of influence of actual network flow over the induced probe packets. 
These probe packets are small in size and won't add much extra load on network. Using the method kalman filtering measuring the available bandwidth is successful and gave good results in their experiments.

Giovanna. C [5] has proposed a receiver driven interest control protocol where it is joint effort of interest control and interest shaping. Interest control protocol is applied to regulate the interest sending rate and set the optimal re-interest timer when there is data packet loss. There is virtual interest queue at each output interface of every router. A credit counter is maintained which increments for data flow and decrements for interest flow. For non-bottleneck flows interest is sent directly and for bottleneck flows interest is inserted in drop tail FIFO queue. Yaogong. W [6] has proposed hop by hop interest shaper where interest shaper runs at each node, shaping the interest in both directions. If the incoming packets are data packets then they are directly placed on L2 queue and if the incoming packets are interests then these interests are placed in interest shaper, then later they move to L2 Queue. If there is overload of interest packets which can't be placed in interest shaper are dropped and NACK is sent back to consumer for adjustment. Simple AIMD mechanism is used at consumer side and this approach is simulated in ndnSIM. There is another extended hop by hop interest shaping approach [7] where the in-network nodes sends the explicit control packets periodically which specifies the maximum sending data rate for every consumer on the path. Based on this explicit rate consumers adjust the interest sending rate, if there is overload of interests then interests are delayed in network routers so that there won't be any congestion when data packets arrive. There is another explicit congestion control approach proposed by Yongmao. R [8] where this approach measures the interest queue length at each in-network node and if the interest queue length exceeds the threshold then that node sends NACK with three different types, NACK overload for multiplicative decrease of interest sending rate, NACK busy for additive increase of interests and NACK free for multiplicative increase if the network path is free. There is a new transport layer design for named data networks [9] interest retransmission timer is introduced for packet loss and self-regulating interest rate control is introduced with simple AIMD mechanism.

In [10] authors have proposed random early marking as explicit congestion control where each and every outgoing data packet is mark explicitly so that the consumer can be intimated about congestion, if there is burst of interests and these interests are stored temporarily and are delayed so that there must be no loss in returning data, if delay of interest does not work then AIMD is used. In [11] authors have proposed network assisted congestion control where reduce sending rate (RSR) message is sent towards downstream routers and process repeats until it hits the consumer.

In [12] authors have proposed IPv6 based smart home using Arduino, but since pure ICN implementation does not require IP based networking and ICN is ready for name base lookup and forwarding so in future there will be not need of IP based network, and ICN is readily scalable in terms of naming convention, also with the help of best caching policy [15] fastest name lookup and fast object storage are retrieval is achieved.

\section{A. Single Path MIRCC}

Multipath-aware ICN rate based congestion control (MIRCC) logic is built upon the ICN architecture based on NDN or CCNx library. In MIRCC a consumer initiates the communication by sending the interests towards producer. On reception of interest producer sends back the data to consumer which also carries the rate value in data packet header which is Max Rate determined by the MIRCC logic. Each hop forwarder forwards the data message back towards the consumer in reverse path determined by the PIT entries. Here each forwarder preserves the data rate $R(t)$ of its associated link as shown in figure 1 . When data packet arrives at in-network forwarder, it compares the data rate in packet header with the available data rate of the adjacent link. If the data rate in the packet header is less than associated link data rate then forwarder stamps the new data rate in that header which is the minimum. In this way forwarder associated with a bottleneck link will be the last one to update the data rate value in the data packet header travelling in reverse path towards consumer. Each link $R(t)$ calculation also gives feedback to the consumer regarding new interest sending rate, if the in-network links are overloaded then the $R(t)$ is stamped with reduced value and is the link is under loaded then $R(t)$ is stamped with higher value. MIRCC also applies interest shaping mechanism at each link which controls the overloading of interest packets which results in control of returning data packets. Overloaded interests which can't be placed in queue are dropped and a NACK is sent towards consumer indicating there is congestion, which in turn consumer will resend the interest without waiting for timeout.

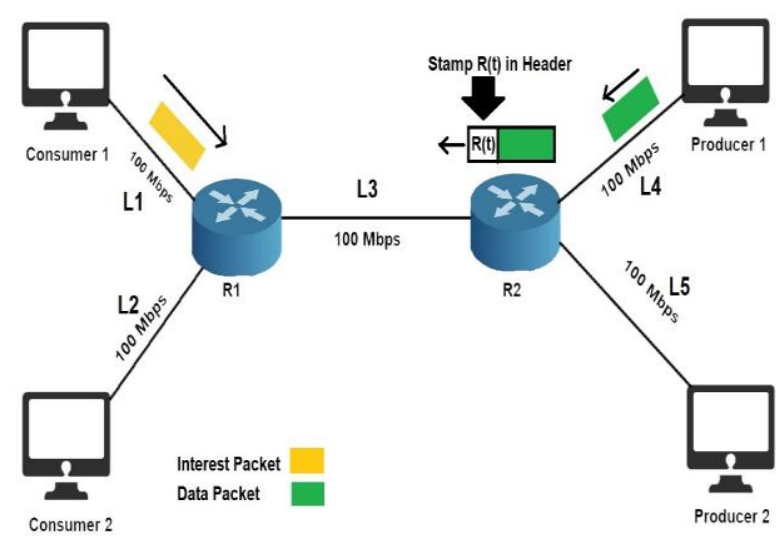

Fig. 1.Single Path MIRCC.

\section{B. Limitations in Single Path MIRCC}

MIRCC considers the bottleneck link with fixed capacity but in actual networking scenarios there are wireless links also present, these wireless links have variable capacity of link bandwidth and tend to change over time. It is expected that MIRCC will perform same as TCP/IP networks when the wireless link capacity is minimum. To solve this problem there is need of link capacity estimation for wireless links which can be achieved by using kalman filter. 


\section{PROPOSED ARCHITECTURE}

In order to overcome the limitation we came up with modified architecture which is called as modified single path MIRCC to estimate available wireless link bandwidth periodically using kalman filter. In this new architecture, wireless links are considered with variable link capacity and to know actual link bandwidth each in-network node calculates adjacent link capacity using kalman filter. This architecture has advantage over MIRCC because it works with both wired as well as wireless networks with low/no packet drops and low latency.

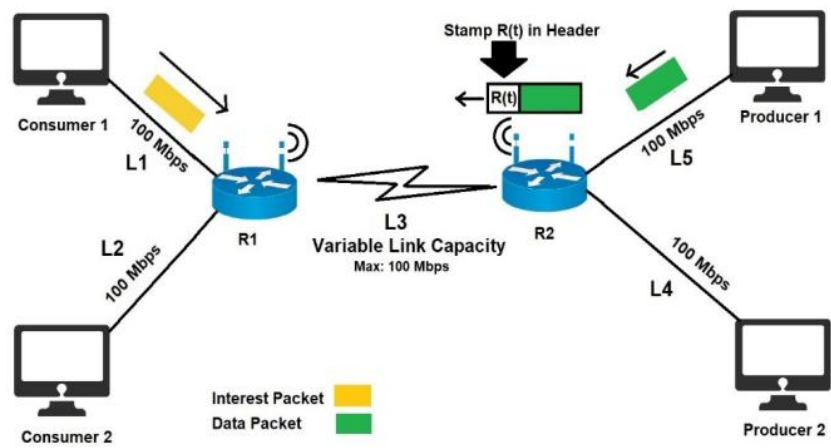

Fig. 2. Modified Single Path MIRCC.

In figure 2 L3 is the bottleneck link with variable link capacity, to be assumed maximum upto100 Mbps. Consumer 1 sends the interest towards producer 1 , which reaches the producer via router $\mathrm{R} 1$ and $\mathrm{R} 2$. On reception of interest producer 1 responds back with data packet with initial data rate (calculated by MIRCC logic) stamped in packet header. $\mathrm{R} 2$ estimates available link capacity of L3 using kalman filter, if the available link capacity of L3 is less than $R(t)$ value in header then it stamps the new $R(t)$ in the data packet header. When consumer receives this data packet then it adjusts its interest sending rate according to new $R(t)$. In this way congestion can be evaded proactively and consumer can be intimated with the actual sending data rate whereas in MIRCC if bottleneck link capacity fall down then consumer would continue to send interest with false data rate and resulting in interests and data packets overload at R1 and R2, which results in loss of packets and lots of delays are expected.

\section{SIMULATION SETUP}

Table- I: Simulation parameters for proposed architecture

\begin{tabular}{|c|l|c|}
\hline \multirow{2}{*}{ Sr. No. } & \multicolumn{1}{|c|}{ Simulation Parameters } \\
\cline { 2 - 3 } & \multicolumn{1}{|c|}{ Parameter } & Value \\
\hline 1 & Operating system & Linux Ubuntu 16.04 \\
\hline 2 & NDN SIM Version & 2.6 \\
\hline 3 & Topology Type & 2 \\
\hline 4 & Number of producers & 2 \\
\hline 5 & $\begin{array}{l}\text { Number of consumers } \\
\text { Number of interests sent per } \\
\text { second }\end{array}$ & $1,10,100,500,1000$, \\
\hline
\end{tabular}

\begin{tabular}{|c|l|c|}
\hline \multirow{2}{*}{ Sr. No. } & \multicolumn{2}{|c|}{ Simulation Parameters } \\
\cline { 2 - 3 } & \multicolumn{1}{|c|}{ Parameter } & Value \\
\hline 7 & Forwarding strategy & Best Route \\
\hline 8 & Cache replacement policy & LRU \\
\hline 9 & Propogation Delay & $10 \mathrm{MS}$ \\
\hline 10 & Maximum Bandwidth & $100 \mathrm{Mbps}$ \\
\hline
\end{tabular}

Our new approach is based on NDN architecture and is simulated in NS3 based custom simulator called ndnSIM. Table 1 summarizes the simulation parameters used in the scenario. Here we use NDN 6-node bottleneck congestion topology to evaluate our results.

\section{SIMULATION RESULTS}

The performance of the proposed MIRCC (Modified MIRCC) is evaluated by the simulation performed on the above mentioned setup.

To have a fair comparison existing MIRCC is also simulated with the same setup for the following cases;
A. Data rate
B. Queue occupancy
C. Total flow completion time

\section{A. Data Rate}

We have run the simulation according to parameters in table I and obtained the results as show in figure 3. From the figure 3 we infer that modified MIRCC adopts the new rate quickly than other rate control protocols [18], where in previous rate control protocols there is much oscillation in terms of data rate and modified MIRCC manages to keep the data rate constant under heavy wireless link load scenarios. Also there is use of interest shaper at each forwarder in modified MIRCC which helps to reduce the oscillations in data rate in Modified MIRCC.

\section{B. Queue occupancy}

Simulations are carried out for queue occupancy according to the parameters given in table 1, according to the results obtained as shown in figure 4 , if wireless links are considered in MIRCC then it may not work optimally and it will piggyback false data rate values to consumers. From figure 4 we infer that when wireless links are considered in existing MIRCC then it works as normal end to end congestion control and consumers will keep sending interests in higher amount than expected, this behavior results in overloading of interests and data packets which will result in routers output buffer overflow in the direction of data packets are travelling. Figure 4 shows the queue occupancy considering the bottleneck link with variable capacity, here the routers output buffer overflows in MIRCC and there are packet drops and retransmission, but modified MIRCC works well when there is sudden network overload, 
which manages the congestion by piggybacking the actual available bandwidth in data packets towards consumers and reducing the requests according to new data rate.

\section{Total flow completion time}

As shown in figure 5, according to our simulations we infer that the total flow completion time of modified MIRCC is less than MIRCC because there will be no packet drops and no retransmission in modified MIRCC. In figure 5 existing single path MIRCC takes more time to complete a flow because of packet drops at the time of congestion. Whereas our modified MIRCC takes less time because consumers are sending interests according to the data rate which they receive optimized for wireless links.

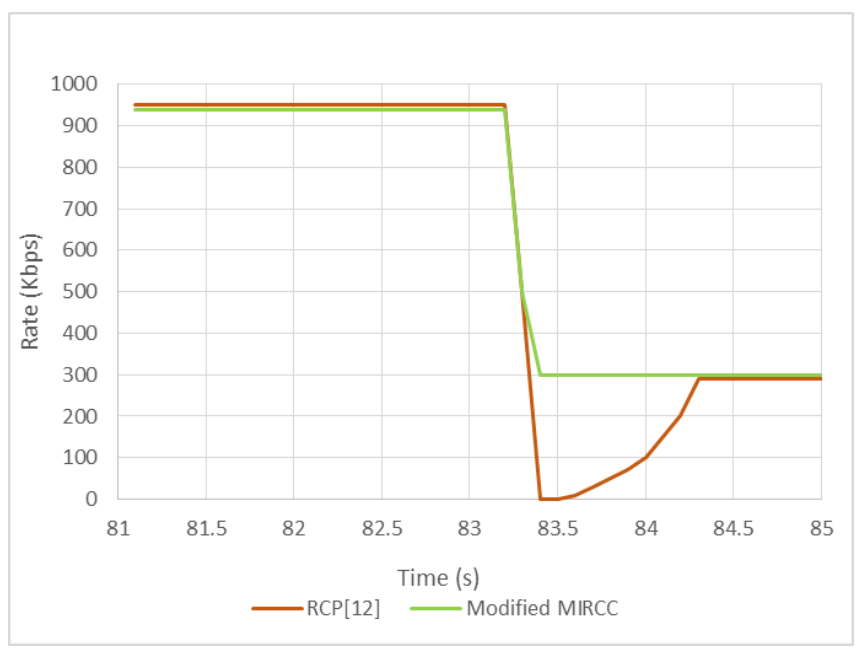

Fig. 3. Data Rate

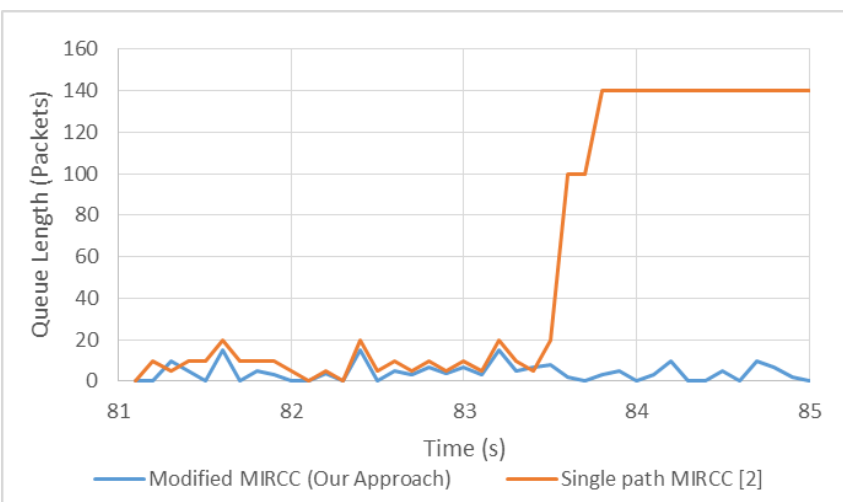

Fig. 4. Queue occupancy

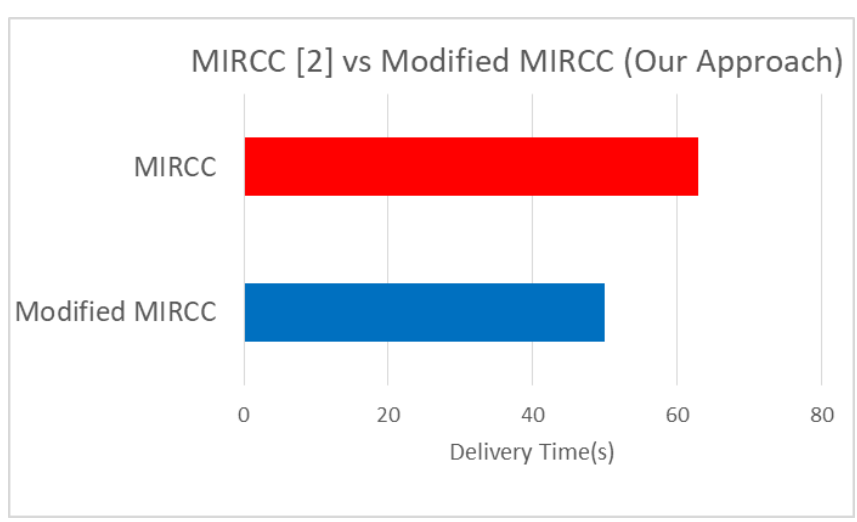

Fig. 5. Total flow completion time

\section{CONCLUSION}

Almost all hop by hop congestion control schemes in ICN assume the link bandwidth as fixed and static, one such kind of approach is MIRCC [2]. This paper demonstrates that MIRCC will not perform the same as expected in wireless scenarios and it converges to normal end to end congestion control. However our approach which is Modified MIRCC considers wired as well as wireless links in scenario and for wireless links dynamic link capacity estimation is carried out. This modified approach results in no output buffer packet drop and low latency. Our future work consist of extending this work in multipath scenario.

\section{FUTURE WORK}

NDN based information centric network is a general architecture which can be deployed in many scenarios, including IoT and sensor networks, where it is very important to manage the energy conservation in the mobile nodes. So to achieve this we need a multi-face and multipath approach to make load balancing strategy for energy conservation [13]. This modified single path MIRCC work will be extended and implemented to multipath approach in future.

\section{REFERENCES}

1. Mahesh R Patil \& Agilandeeswari L. (2019, July) A Role of Routing, Transport and Security Mechanisms in Information Centric Network. In International Journal of Recent Technology and Engineering (IJRTE) (Vol. 8, No. 2S4, pp. 196-203)

2. Ahlgren, B., Hurtig, P., Abrahamsson, H., Grinnemo, K. J., \& Brunstrom, A. (2018, April). ICN congestion control for wireless links. In Wireless Communications and Networking Conference (WCNC), 2018 IEEE (pp. 1-6). IEEE.

3. Mahdian, M., Arianfar, S., Gibson, J., \& Oran, D. (2016, September). MIRCC: Multipath-aware ICN rate-based congestion control. In Proceedings of the 3rd ACM Conference on Information-Centric Networking (pp. 1-10). ACM.

4. Ekelin, S., Nilsson, M., Hartikainen, E., Johnsson, A., Mangs, J. E., Melander, B., \& Bjorkman, M. (2006, April). Real-time measurement of end-to-end available bandwidth using kalman filtering. In 2006 IEEE/IFIP Network Operations and Management Symposium NOMS 2006 (pp. 73-84). IEEE.

5. Carofiglio, G., Gallo, M., \& Muscariello, L. (2012). Joint hop-by-hop and receiver-driven interest control protocol for content-centric networks. ACM SIGCOMM Computer Communication Review, 42(4), 491-496.

6. Wang, Y., Rozhnova, N., Narayanan, A., Oran, D., \& Rhee, I. (2013, August). An improved hop-by-hop interest shaper for congestion control in named data networking. In ACM SIGCOMM Computer Communication Review (Vol. 43, No. 4, pp. 55-60). ACM.

7. Rozhnova, N., \& Fdida, S. (2014, December). An extended Hop-by-hop Interest shaping mechanism for Content-Centric Networking. In IEEE GLOBECOM 2014.

8. Ren, Y., Li, J., Shi, S., Li, L., \& Wang, G. (2016, April). An explicit congestion control algorithm for named data networking. In Computer Communications Workshops (INFOCOM WKSHPS), 2016 IEEE Conference on (pp. 294-299). IEEE.

9. Amadeo, M., Molinaro, A., Campolo, C., Sifalakis, M., \& Tschudin, C. (2014, April). Transport layer design for named data wireless networking. In Computer Communications Workshops (INFOCOM WKSHPS), 2014 IEEE Conference on(pp. 464-469). IEEE.

10. Zhang, F., Zhang, Y., Reznik, A., Liu, H., Qian, C., \& Xu, C. (2014, August). A transport protocol for content-centric networking with explicit congestion control. In Computer Communication and Networks (ICCCN), $2014 \quad 23 r d$ International Conference on (pp. 1-8). IEEE. 
11. Ndikumana, A., Ullah, S., Kamal, R., Thar, K., Kang, H. S., Moon, S. I., \& Hong, C. S. (2015, August). Network-assisted congestion control for information centric networking. In Network Operations and Management Symposium (APNOMS), 2015 17th Asia-Pacific (pp. 464-467). IEEE.

12. Mahesh Patil, Sumathy S., R. Hegadi, IPv6 Enabled Smart Home Using Arduino, In 2016 International Conference on Communications, Information Management and Network Security, Atlantis press 2352-538X.

13. R. Desai, Harish H.S., Raghu H. Mahesh Patil, Novel and Energy Efficient Routing in Wireless Sensor Networks. In National Conference on Knowledge, Innovation in Technology and Engineering, International Journal of Computer Applications (0975 - 8887).

14. Jain, S., Zhang, Y., \& Loguinov, D. (2008, June). Towards experimental evaluation of explicit congestion control. In Quality of Service, 2008. IWQoS 2008. 16th International Workshop on (pp. 121-130). IEEE.

15. Hegadi, R., Kammar, A., \& Budihal, S. (2019, March). Performance Evaluation of In-network Caching: A Core Functionality of Information Centric Networking. In 2019 International Conference on Data Science and Communication (IconDSC) (pp. 1-8). IEEE.

16. Mejri, S., Touati, H., Malouch, N., \& Kamoun, F. (2017, October). Hop-by-Hop Congestion Control for Named Data Networks. In 2017 IEEE/ACS 14th International Conference on Computer Systems and Applications (AICCSA) (pp. 114-119). IEEE.

17. Hayamizu, Y., \& Yamamoto, M. (2015, May). Receiver-driven congestion control for content oriented application with multiple sources. In Communications Quality and Reliability (CQR), 2015 IEEE International Workshop Technical Committee on (pp. 1-6). IEEE.

18. Xia, C., \& Xu, M. (2012, October). RRCP: A Receiver-Driven and Router-Feedback Congestion Control Protocol for ICN. In Networking and Distributed Computing (ICNDC), 2012 Third International Conference on (pp. 77-81). IEEE.

19. Wang, Z., Luo, H., Zhou, H., \& Li, J. (2018). R 2 T: A Rapid and Reliable Hop-by-Hop Transport Mechanism for Information-Centric Networking. IEEE Access, 6, 15311-15325.

20. Zhou, J., Wu, Q., Li, Z., Kaafar, M. A., \& Xie, G. (2015, June). A proactive transport mechanism with explicit congestion notification for NDN. In Communications (ICC), 2015 IEEE International Conference on (pp. 5242-5247). IEEE.

21. Carofiglio, G., Gallo, M., \& Muscariello, L. (2016). Optimal multipath congestion control and request forwarding in information-centric networks: Protocol design and experimentation. Computer Networks, 110, 104-117.

22. Li, C., Xie, R., Huang, T., \& Liu, Y. (2017). Jointly optimal congestion control, forwarding strategy and power control for named-data multihop wireless network. IEEE Access, 5, 1013-1026.

23. Arianfar, S., Nikander, P., Eggert, L., \& Ott, J. (2010). Contug: A receiver-driven transport protocol for content-centric networks. Under submission.

24. Jacobson, V., Smetters, D. K., Thornton, J. D., Plass, M. F., Briggs, N. H., \& Braynard, R. L. (2009, December). Networking named content. In Proceedings of the 5th international conference on Emerging networking experiments and technologies (pp. 1-12). ACM.

25. Yeh, E., Ho, T., Cui, Y., Liu, R., Burd, M., \& Leong, D. (2013). Forwarding, caching and congestion control in named data networks. arXiv preprint arXiv: 1310.5569.

26. Dukkipati, N., \& McKeown, N. (2006). Why flow-completion time is the right metric for congestion control. ACM SIGCOMM Computer Communication Review, 36(1), 59-62.

27. Jose, L., Yan, L., Alizadeh, M., Varghese, G., McKeown, N., \& Katti, S. (2015, November). High speed networks need proactive congestion control. In Proceedings of the 14th ACM Workshop on Hot Topics in Networks (p. 14). ACM.

28. Dukkipati, N. (2008). Rate Control Protocol (RCP): Congestion control to make flows complete quickly. Stanford University.

29. Ahlgren, B., Dannewitz, C., Imbrenda, C., Kutscher, D., \& Ohlman, B. (2012). A survey of information-centric networking. IEEE Communications Magazine, 50(7).

30. Tanaka, D., \& Kawarasaki, M. (2016, June). Congestion control in named data networking. In Local and Metropolitan Area Networks (LANMAN), 2016 IEEE International Symposium on (pp. 1-6). IEEE.

31. Saino, L., Cocora, C., \& Pavlou, G. (2013, June). Cctcp: A scalable receiver-driven congestion control protocol for content centric networking. In Communications (ICC), 2013 IEEE International Conference on (pp. 3775-3780). IEEE.

\section{AUTHORS PROFILE}

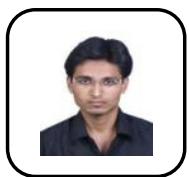

Mahesh R. Patil received his M. Tech in Information Technology (Networking) from VIT University Vellore India in Aug 2016, later same year he worked as Assistant Professor at Walchand Institute of Technology Solapur India for one year and is currently a research scholar in VIT University Vellore India. His research interests include computer networks and future Internet technologies with a focus on Information-Centric Networking and Internet of Things.

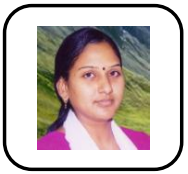

L. Agilandeeswari is working as an Associate Professor in the School of Information Technology and Engineering (SITE) at VIT University, Vellore. She received her B. Tech in Information Technology and M.E in Computer Science and Engineering from the Anna University with honors during 2005 and 2009, respectively. She completed her PhD at the VIT University, Vellore with good number of publications indexed by Scopus and Thomson Reuters with an impact factor of $>4$. She has also more than 20 international and national conference publications. She has around 11 years of teaching experience. She is a life time member in Computer Society of India. Her areas of interests include image and video watermarking, Information Centric Networks, Image and video processing, neural networks, and Data mining. 